\title{
Уточненное борновское приближение для рассеяния электромагнитной волны наночастицами
}

\author{
$\underline{\text { А.С. Берёза* }}^{*}$ Д.А. Шапиро \\ Институт автоматики и электрометрии СО РАН \\ Новосибирский Государственный Университет \\ "E-mail: alex.bereza2010@yandex.ru
}

DOI: 10.31868/RFL2020.29

В настоящее время с развитием численных методов исследования и применением электронных вычислительных машин круг решаемых задач значительно расширился. В отличие от аналитических решений, численные методы обладают большей универсальностью и позволяют получить решение с заданной степенью точности, тем не менее, аналитическое решение по-прежнему остается исключительно ценным для выявления общих теоретических закономерностей. Одним из способов получения аналитического решения является борновское приближение, суть которого в том, чтобы для слабого рассеивателя вместо полного поля внутри рассеивающего потенциала использовать падающее поле. Однако, традиционный борновский ряд не применим к системам с резкой границей, поскольку не позволяет точно учесть граничные условия.

Целью данной работы является построение модифицированного борновского приближения для задачи рассеяния электромагнитной волны на нанообъектах. Смысл в том, чтобы разбить имеющуюся задачу для возмущенной и невозмущенной среды: для последней строится специальная функция Грина [1], что позволяет автоматически учесть условия на границе. После выводятся интегральные соотношения, связывающие внешнее поле и поле внутри рассеивателя, и далее методом последовательных приближений получается решение в виде борновского ряда. Такое борновское приближение уже точно учитывает все граничные условия.

Для случая s- и p- поляризаций была построена функция Грина и получена первая борновская поправка к рассеянному полю, а также рекуррентные соотношения для нахождения последующих поправок. Также проанализирована сходимость борновского ряда, и получены критерии применимости. Метод сверен с двумя независимыми численными решениями: методом точечных диполей [2] и методом граничных элементов [3]. Показано, что первое борновское приближения подходит для определения качественного характера рассеяния, второе и последующие могут быть использованы для описания его точных количественных характеристик.

\section{Литература}

[1] A.S. Bereza, L.L. Frumin, et al, EPL 127, 20002-p1 - 20002-p5 (2019)

[2] A.S. Bereza, A.V. Nemykin, et al, Phys. Rev. A 95, 063839-1 - 063839-6 (2017)

[3] O.V. Belai, L.L. Frumin, et al, EPL 97, 10007-10016 (2012). 